\section{Membranbildung bei kernlosen, wachsenden und nicht wachsenden Teilen von}

\section{Acetabularia mediterranea}

\author{
Von Günther Werz *
}

Aus dem Max-Planck-Institut für Meeresbiologie, Wilhelmhaven, Abt. Prof. Dr. J. Hämmerting

(Z. Naturforschg. 12 b, 739-740 [1957]; eingeg. am 22. August 1957)

Kernlose Vorderstücke von kurz vor der Hutbildung stehenden Acetabularien besitzen bekanntlich ausgeprägtes mit Membranbildung verbundenes Formbildungsvermögen. Dieses kann - je nach Länge der Teile und Zustand der Kultur - außer zur Stielbildung und Bildung von Wirteln bis zur Bildung großer Hüte führen. Dagegen besitzen kernlose Hinterstücke kein Wuchsvermögen ${ }^{1}$. Untersuchungen, ob in den Hinterstücken das Substanzgleichgewicht erhalten bleibt oder ob eine Substanzverminderung erfolgt, ergaben das überraschende Resultat, daß im Gegenteil auch in diesen Teilen eine Substanzproduktion stattfindet, wenn auch in erheblich geringerem Maße als bei den Vorderstücken ${ }^{2 a, b, c}$. Als Beispiele dafür seien angeführt: die Vermehrung des Gesamt-Eiweiß-NGehaltes (WERZ in 1. c. $\left.^{2 b}, \mathrm{c}\right)$, und zwar sowohl der Cytoplosma- wie der Chloroplastenproteine ${ }^{3}$, die Vermehrung des Polyphosphatgehaltes ${ }^{4}$, des Gesamt-PGehaltes (Werz in l. c. ${ }^{2 a, b}$ ) und die Vermehrung des Kohlenhydratgehaltes ${ }^{5}$. Nach diesen Befunden erhob sich die Frage, ob die Hinterstücke deshalb keine Stielneubildung besitzen, weil sie im Gegensatz zu den Vorderstücken unfähig zur Membranbildung sind. Die im folgenden mitgeteilten Untersuchungen zeigten jedoch, daß eine andere Situation vorliegt.

Die Versuche wurden an kernlosen Vorderstücken und Hinterstücken von ca. $50 \mathrm{~mm}$ langen, hutlosen Ausgangspflanzen von Acetabularia mediterranea durchgeführt. Sie stammten von den gleichen Pflanzen, ihre Ausgangslängen betrugen $11 \mathrm{~mm}$ (Nachzucht $m \mathrm{f}_{4}$ v. 21. 11. 1954; hellgestellt 27. 7. 1955) ; über Aufzucht- und Kulturbedingungen s. Н̈̈мMERLING ${ }^{6}$ und $\mathrm{BETH}^{7}$. Die Versuchsteile wurden in Erdschreiber bei $12 \mathrm{Stdn}$. täglicher Beleuchtung mit ca. $2500 \mathrm{Lux}$ gehalten; die Versuchstemperatur betrug ca. $22^{\circ}$ C. Es wurde absichtlich eine Nachzucht verwendet, deren Vorderstücke weniger als $2 \%$ Hüte bildeten. Die Membrangewichte der praktisch also nur Stiel bildenden Vorderstücke wurden mit den nicht stielbildenden Hinterstücken verglichen. Diesen fehlte nur das Rhizoid mit 0,5-1,0 mm Stiel. Die Membrangewichte wurden nach Fixierung der Teile in 20-proz. Trichloressigsäure, je 18-stdg. Extraktion mit Trypsin ( $p_{\mathrm{H}} 7,2$; $\left.37^{\circ} \mathrm{C}\right)$ und Pepsin $\left(p_{\mathrm{H}} 2,0 ; 37^{\circ} \mathrm{C}\right)$ und anschließender Behandlung mit 10-proz. KOH (3 Stdn. bei $50^{\circ} \mathrm{C}$ ), Waschen in Alkohol, Äther und Trocknen im Vakuum bestimmt. Es

* Die Versuche wurden mit personeller und sachlicher Unterstützung durch die Deutsche Forschungs. ge me in s ch a f $\mathrm{t}$ ausgeführt.

1 J. Hämmerling, Biol. Zbl. 52, 42 [1932]; Arch. Entwicklungsmech. 132, 424 [1934].

2 J. Hämmerling, a) Int. Rev. Cytology 2, 475 [1953]; b) 8 ème Congr. Internat. de Bot., C. R. Séances et Rapp. et Communic. déposés lors du Congr. dans la Section 10, 87 [1957]; c) Biol. Zbl. 74, 545 [1955]. wurden jeweils 40 Teile gemessen, und zwar in getrennten Bestimmungen zu je 10 Teilen. Für beide Serien war der 0-Tag der 2. Tag nach der Amputation.

Sehr wahrscheinlich besteht die Membran nicht aus reiner Cellulose ${ }^{8}$; wir verwenden deshalb nur den allgemeinen Ausdruck Membran und nicht Cellulosemembran und lassen auch dahingestellt, ob die von kernlosen Teilen gebildete Mem. bran, insbesondere die der Hinterstücke, von gleichem chemischem und strukturellem Aufbau ist wie die Membran der kernhaltigen Pflanzen.

Die Vorderstücke wiesen eine Zunahme ihrer Stiellängen um durchschnittlich $6 \mathrm{~mm}$ auf (Abb. 1). Die Hauptzunahme durch Spitzenwachstum war nach ca.

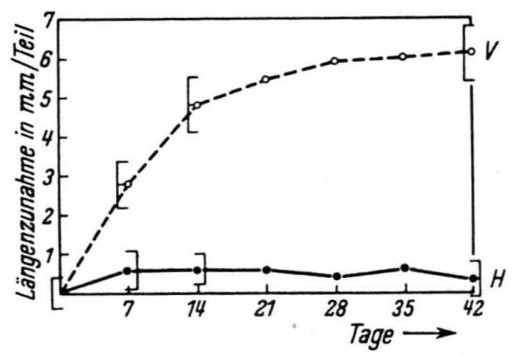

Abb. 1. Stiel-Längenzunahmen (in $\mathrm{mm} /$ Teil) bei kernlosen Vorderstücken $(V)$ und Hinterstücken $(\mathrm{H})$. Ausgangslängen je $11 \mathrm{~mm}$. Mittelwerte \pm 3 -facher mittlerer Fehler. $n=$ jeweils $\sim 50$.

14 Tagen beendet. Dies entspricht der allgemeinen Regel, daß Teile, die nur Stiel bilden, ihr Wachstum nach rund zwei Wochen beenden. Die danach erfolgenden, sehr geringen Längenzunahmen sind mindestens teilweise auf sekundäre Streckung zurückzuführen, wie sie an den sich vergrößernden Abständen zwischen zwei Wirtel-Ansatzstellen abgelesen werden kann ${ }^{9}$. Die Membranbildung der Vorderstücke war im wesentlichen ebenfalls mit dem ungefähr 14. Tage beendet (Abb. 2). Bei den fast nicht wachsenden Hinterstücken (Abb. 1) ergab sich, daß diese ihr Membrangewicht ebenfalls erheblich vermehrten (Abb. 2), wobei die Hauptzunahme wiederum in den ersten beiden Wochen erfolgte. $\mathrm{Ob}$ die Membranvermehrung sogar stärker war als bei den Vorderstücken, ließe sich nur durch umfangreicheres Material entscheiden, jedenfalls war sie bei der hier verwendeten Nachzucht anscheinend nicht geringer. (Würden Vorderstücke verwendet werden, die in hohem Prozentsatz Hüte bilden, so wäre ihre Membranbildung sicher wesentlich höher als bei nur stielbildenden Vorderstücken und damit auch höher als bei den zugehörigen Hinterstücken.) Das AusgangsMembrangewicht der Hinterstücke war mit $46 \mu \mathrm{g} /$ Teil

${ }^{3}$ H. Clauss , unveröff.

${ }^{4}$ H. Sтich, Z. Naturforschg. 8 b, 36 [1953]; 10 b, 281 [1955].

5 K. KECK, unveröff.

6 J. Hämmerling, Arch. Protistenkunde 97, 7 [1944].

7 K. Beth, Z. Naturforschg. 8 b, 334 [1953].

8 G. WERZ, unveröff.

9 J. Hämmerling, mündl. Mitteil. 
wegen der großen Dicke der alten Membran wesentlich höher als das der Vorderstücke mit ihrer besonders ganz vorne eben gebildeten, dünnen Membran (11,5 $\mu \mathrm{g} /$ Teil). Dies gilt übrigens auch für den Eiweißgehalt, der bei Hinterstücken über dem der Vorderstücke liegt ${ }^{8}$.

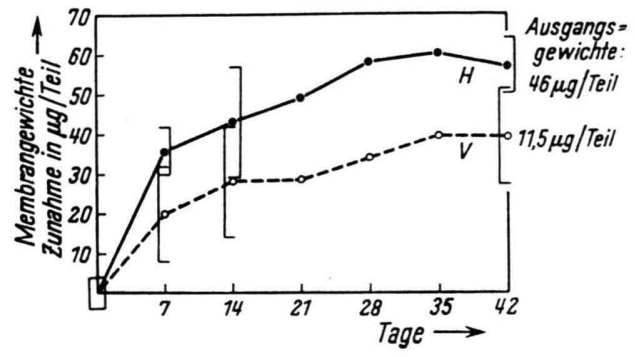

Abb. 2. Zunahmen der Membrangewichte bei kernlosen Vorderstücken $(V)$ und Hinterstücken $(H)$. Mittelwerte \pm 3 -facher mittlerer Fehler; $n=$ jeweils 40; Ausgangsgewichte: $\mathrm{V}=11,5 \mu \mathrm{g} /$ Teil, $\mathrm{H}=46 \mu \mathrm{g} /$ Teil.

Bei den nichtwachsenden Hinterstücken ist die allmähliche Membranverdickung schon optisch feststellbar (Abb. 3). Die Gewichtsbestimmungen zeigen, daß eine sehr erhebliche Zunahme des Trockengewichtes vorliegt, die neue Membran also nicht etwa durch Wasserimbibition stark aufgequollen wird. Die großen Schwankungen der Membrangewichte sind natürlicher Art. Auch unter möglichst gleichartigen Aufzuchtbedingungen sind die Membranen sehr verschieden dick.

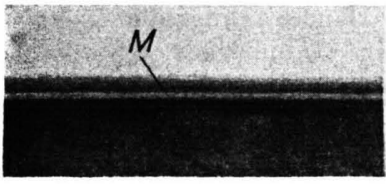

a

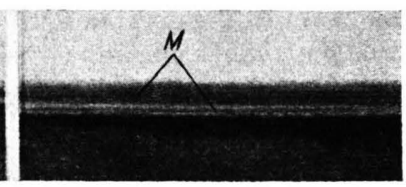

b
Abb. 3. Membrandicken desselben kernlosen Hinterstückes am Tage 0 (a) und nach 40 Tagen Licht (b). M Membran (schärfere Kontraste durch schwache Anfärbung der Membran durch Trypaflavin in vivo; ca. 75 -fach). Ob die in Abb. 3 b erkennbare Zweischichtigkeit der Membran real ist oder lediglich durch optische Effekte vorgetäuscht wird, ließ sich noch nicht entscheiden.

Die nach Entfernung des Kernes wachsenden und nicht wachsenden Teile unterscheiden sich also nicht in der Fähigkeit oder Unfähigkeit zur Membransynthese. Beide Systeme bildeten vielmehr neue Membran, die nur stielbildenden Vorderstücke sogar in nicht stärkerem Maße. Der Unterschied zwischen Vorder- und Hinterstücken liegt vielmehr in der Art der Membranbildung. Die nichtwachsenden Hinterstücke bilden im wesentlichen neue Membran durch Apposition (Abb. 3), die Vorderstücke durch Intussuszeption, und zwar in Form einer longitudinalen Anlagerung an der Spitze. Völlig unfähig zur Intussuszeption sind aber auch die nichtwachsenden Hinterstücke nicht. An ihren Schnittflächen wird regelmäßig die Abschlußmembran intussuszeptionell gebildet, auch bei mehrfachen Amputationen, nur führt dies nicht oder nur selten und dann sehr gering zu Spitzenwachstum. Andererseits weisen die Vorderstücke auch appositionelle Membranbildung auf, wie daraus hervorgeht, daß die anfänglich sehr dünne neue Membran (wie bei der Stielbildung kernhaltiger Teile) mit der Zeit dicker wird.

Die weitgehende Unfähigkeit der nichtwachsenden Teile zur intussuszeptionellen Membranbildung mit Spitzenwachstum kann nicht auf einen allgemeinen strukturellen Unterschied zwischen dem Vorder- und Hinterende der Zelle zurückgeführt werden, sondern muß auf dem Mangel eines hierfür spezifischen Faktors beruhen. Dies geht besonders aus Versuchen von J. und Ch. Hämmerling ${ }^{10}$ hervor, bei denen u. a. gezeigt werden konnte, daß dieser Faktor unter der sogar in Dunkelheit erfolgenden Wirkung des Zellkernes auch bei sonst nichtwachsenden Teilen intussuszeptionelles Membranwachstum anregen kann, sowie daraus, daß auch die Hinterstücke nach Transplantation eines kernhaltigen Rhizoides ${ }^{1}$ oder der Implantation eines in vivo isolierten Kernes ${ }^{2 \mathrm{c}}$ normal auswachsen können. Erst wenn dieser Faktor, der vorläufig zu dem pauschalen Begriff „morphogenetische Substanzen“ gerechnet werden kann ${ }^{2 a, b}$ in einem kernlosen Teilstück in ausreichender Menge vorhanden ist, kommt es zur Stielneubildung und damit zu normalem Wachstum. Die in der Einleitung aufgeführte Nettosynthese verschiedenster Substanzen, darunter vor allem von Proteinen - auch in den nicht wachsenden Hinterstücken - hatte bereits gezeigt, daß die Bildung dieser Stoffe nicht hinreichende Bedingung für das Zellwachstum ist (vgl. l. c. $\left.{ }^{2 b, c}\right)$. Die hier geschilderten Befunde geben einen ersten Einblick, warum es so ist. Die Stielneubildung wiederum hat eine weitgehende Rückwirkung auf den Grad der Proteinsynthese, wie an anderer Stelle gezeigt werden soll.

10 Cit. in J. Hämmerling u. H. Stich, Z. Naturforschg. 11 b. 162 [1956]. 